ANCAs. Antigen discovery to determine the targets of these recombinant plasmablast antibodies is ongoing.

Conclusions: Plasmablasts from patients with GPA in the RAVE trial were sequenced and antibody repertoires were generated. Clonal families of plasmablasts, including those expressing antibodies possessing shared CDR sequences across multiple patients, were identified and recombinantly expressed. None of the 24 antibodies bound to PR3, suggesting these are not ANCAs and are potentially novel autoantibodies. Identification of the antigen targets of these antibodies is ongoing.

Acknowledgements: The Immune Tolerance Network

Training Program in Adult and Pediatric Rheumatology 2T32AR050942-11

Disclosure of Interest: None declared

DOI: 10.1136/annrheumdis-2017-eular.3261

\section{SAT0018 EFFECTS OF ANTI-TNF ALPHA THERAPY ON B CELLS IN RHEUMATOID ARTHRITIS (RA) PATIENTS}

O. Pala $^{1}$, A. Diaz ${ }^{2}$, B. Aubourg ${ }^{1}$, E. Greidinger ${ }^{1}$, B.B. Blomberg ${ }^{2}$, D. Frasca ${ }^{2}$ ${ }^{1}$ Rheumatology; ${ }^{2}$ Microbiology\&Immunology, University of Miami, Miami, United States

Background: Our laboratory has previously characterized defects in humoral B cell responses of aged mice and humans. These defects include: the reduction in activation-induced cytidine deaminase (AID), required for the generation of optimal antibody responses, and the reduction in the percentage/number of the subset of switched memory B cells (1). AID and switched memory B cells have been proposed to be effective predictive biomarkers of vaccine responses (2). Moreover, we have shown that aging is characterized by increased systemic inflammation which induces intrinsic B cell inflammation, measured by intracellular (ic) TNF- $\alpha$, and this significantly decreases the capacity of the same B cells to make protective antibodies in response to vaccination (3). Other marker of $B$ cell intrinsic inflammation is micro-RNA (miR) expression, particularly miR-16 and miR-155, which is increased in elderly $B$ cells and negatively correlated with $B$ cell function (4)

Objectives: Our goal for this study was to evaluate B cell phenotype and function in RA patients treated Methotrexate (MTX), alone or together with anti-TNF- $\alpha$. We hypothesized that patients treated with anti-TNF- $\alpha$ will show improved B cell function due to reduction in icTNF- $\alpha$.

Methods: We recruited 9 RA patients, 5 patients on MTX and 4 on MTX/anti-TNF$\alpha$. We measured the relevant $B$ cell subsets in blood (Naïve, switched memory, IgM memory and late memory) by flow cytometry. Staining was performed with antibodies specific for CD19, CD27 and IgD. In addition, we isolated blood B cells using magnetic beads, and measured the expression of miR-16 and miR-155 on blood B cells by qPCR.

Results: Preliminary data showed that the percentages of switched memory (IgD-CD27+) B cells are significantly higher $(\mathrm{p}<0.003)$ in patients undergoing MTX/anti-TNF- $\alpha$ therapy. We also observed a significant decrease in naïve B cell percentages $(p<0.028)$. Preliminary results also showed a decrease in the mRNA expression of both miR-16 and miR-155 in patients on combination therapy compared to MTX alone.

Conclusions: These results support the hypothesis that therapy with anti-TNF- $\alpha$ is beneficial for improving $B$ cell function in RA patients, as compared to MTX therapy alone. Future experiments will seek to evaluate intrinsic B cell TNF levels in these patients and correlate them with measurements of $B$ cell function. Treatment with anti-TNF- $\alpha$ may be able to block the excessive amounts of systemic TNF- $\alpha$ and in turn B cell intrinsic TNF- $\alpha$ which could improve the antibody responses and the risk of infections in RA patients undergoing therapy. References:

[1] Frasca, D., and B. B. Blomberg. 2013. Age effects on mouse and human B cells. Immunol Res. 57(1-3): 354-360.

[2] Frasca, D., A. Diaz, M. Romero, M. Phillips, N. V. Mendez, A. M. Landin, and B. B. Blomberg. 2012. Unique biomarkers for B-cell function predict the serum response to pandemic H1N1 influenza vaccine. Int Immunol. 24(3): 175-182.

[3] Frasca, D., M. Romero, A. Diaz, S. Alter-Wolf, M. Ratliff, A. M. Landin, R. L. Riley, and B. B. Blomberg. 2012. A Molecular Mechanism for TNF-alphaMediated Downregulation of B Cell Responses. J Immunol 188: 279-286.

[4] Frasca, D., A. Diaz, M. Romero, F. Ferraci, and B. B. Blomberg. 2015. MicroRNAs miR-155 and miR-16 Decrease AID and E47 in B Cells from Elderly Individuals. J. Immunol.195 (5): 2134-2140.

Disclosure of Interest: None declared

DOI: 10.1136/annrheumdis-2017-eular.4331

\section{SAT0019 ESTROGEN INFLUENCES THE SIALYLATION PROFILE AND INFLAMMATORY PROPERTIES OF ANTIBODIES - A POTENTIAL EXPLANATION FOR THE SEX DIFFERENCES AND INCREASED RISK FOR RA IN POSTMENOPAUSAL WOMEN}

C. Engdahl $^{1,2}$, J. Raufer ${ }^{1}$, U. Harre ${ }^{1}$, A. Bondt ${ }^{3}$, R. Pfeifle ${ }^{1,4}$, G. Krönke ${ }^{4,5}$, H.U. Scherer ${ }^{6}$, H. Forsblad ${ }^{2,7}$, G. Schett ${ }^{1} .{ }^{1}$ Department of Internal Medicin 3, Clinical Immunolgy, Erlangen, Germany; ${ }^{2}$ Department of Rhemtology and Inflammation reserch, CBAR, Institue of Medicine, Gothenburg, Sweden; ${ }^{3}$ Department of Rheumatology, Lediden University Medical Center, Leiden,
Netherlands: ${ }^{4}$ Nikolaus-Fiebiger Center for Molecular Medicine, FAU Erlangen-Nuremberg, Erlangen; ${ }^{5}$ Department of Internal Medicin 3, Clinical Immunolgy, Gothenburg, Germany; ${ }^{6}$ Department of Rheumatology, Lediden University Medical Center, Leiden, Netherlands; ${ }^{7}$ Department of Public Health and Clinical Medicine, Rheumatology, Umeå University, Umeå, Sweden

Background: Rheumatoid arthritis preferentially affects women. RA has its peak over 50 years coincidencing with the decrease in sex hormones in menopause. Recently, the transition from asymptomatic autoimmunity to RA has been shown to essentially depend on the glycosylation status of antibodies affecting the binding affinity to Fc gamma receptors ${ }^{1}$. Hence a decrease in the sialylation of antibodies resulting from a decrease in the activity of the sialylation enzyme $\beta$-galactoside $\alpha 2,6$-sialylltransferase (St6Gal1) was shown to trigger the onset of RA.

Objectives: To test whether estrogen influences the glycosylation status of antibodies and St6Gal1 expression explaining why postmenopausal women are particularly prone to develop RA

Methods: In the experimental part we tested the influence of estrogen on antibody glycosylation and St6Gal1 expression. Ovariectomized mice, which were either left without estrogen supplementation or were supplemented with estrogen (hormone replacement), were immunized with ovalbumin (OVA) to induce antibody production. Immunoglobulin G ( $\mathrm{lgG}$ ) levels were analyzed by ELISA and the glycosylation of the Fc-part of total and OVA-specific IgG was determined by lectin ELISA and MALDI-TOF, respectively. St6Gal1 expression in plasma cells was determined by RT-PCR and FACS. Inhuman part we measured the effects of estrogen treatment on autoantibody levels and IgG glycosylation in a cohort of postmenopausal RA patients over 2 years ${ }^{2}$.

Results: Ovariectomy and loss of estrogens was associated with a lower sialylation of OVA-specific IgG. Conversely estrogen treatment significantly increased the sialylation level of newly formed OVA-specific and totals IgG as well as enhanced the expression of St6Gal1 enzyme in plasma cells suggesting a shift towards an anti-inflammatory pattern of IgG. These results were confirmed with estrogen treated postmenopausal RA patients showing that hormone replacement therapy significantly increased antibody glycosylation, while in a control RA population not exposed to estrogens no such increase in sialylation of IgG was found. Estrogens however, did not influence the CCP autoantibody levels. Conclusions: These findings indicate that estrogen regulates St6Ga1 and increases the glycosylation of IgG. Lack of estrogen decreases lgG glycosylation and results in pro-inflammatory properties of $\lg \mathrm{G}$ which may explain the increase prevalence of RA in postmenopausal women.

\section{References:}

[1] Pfeifle et al. Regulation of autoantibody activity by the IL-23- $T_{H} 17$ axis determines the onset of autoimmune disease. Nat Immunol. 2017 Jan;18(1):104113.

[2] D'Elia HF, et al. Influence of hormone replacement therapy on disease progression and bone mineral density in rheumatoid arthritis. J Rheumatol. 2003 Jul;30(7):1456-63.

Disclosure of Interest: None declared

DOI: 10.1136/annrheumdis-2017-eular.6287

\section{SAT0020 THE CITRULLINOME IN TISSUE AND BIOFLUIDS OF HUMAN AND MOUSE ORIGIN}

T.B.G. Poulsen ${ }^{1}$, D. Damgaard ${ }^{2}$, T.B. Bennike ${ }^{1}$, M.K. Meyer ${ }^{3}$, K.J. Elbæk ${ }^{1}$, V. Andersen ${ }^{4}$, S. Birkelund ${ }^{1}$, C.H. Nielsen ${ }^{2}$, A. Stensballe ${ }^{1} .{ }^{1}$ Department of Health Science and Technology, Aalborg University, Aalborg; ${ }^{2}$ Center for Rheumatology and Spine Diseases, Copenhagen University Hospital, Rigshospitalet, Institute for Inflammation Research, Rigshospitalet, Institute for Inflammation Research, Copenhagen: ${ }^{3}$ Department of Rheumatology, North Denmark Regional Hospital, Hjørring; ${ }^{4}$ IRS-Center Sonderjylland, Hospital of Southern Jutland, Aabenraa, Denmark

Background: Protein citrullination is fundamental to several essential processes in apoptosis and antimicrobial defense, however, also linked to multiple pathogenic endpoints. This post-translational modification (PTM), by conversion of arginine to citrulline residues, is mediated by peptidylarginine deiminase (PAD) enzymes found in specific cells and tissues. In polymorphonuclear cells (PMNs) these enzymes enable NETosis, a specialized form of programmed necrosis and the formation of NETs (neutrophil extracellular traps). Also, these enzymes are expressed in the synovium of patients with rheumatoid arthritis (RA) thereby triggering the production of autoantibodies against citrullinated proteins (ACPAs). Objectives: Our objective was to optimize methodology for characterization of this PTM and determine the citrullinome in tissue and biofluids of human and mouse origin in clinical relation to rheumatoid arthritis (RA), osteoarthritis (OA), Spondyloarthritis (SpA) as well as presence of ACAPs and NETs

Methods: Synovial fluid (SF) and plasma was collected from patients diagnosed with RA, OA, SpA $(n=120)$. Inflammation levels patients were characterized with plasma C-reactive protein (CRP), and circulating anti-CCP levels as well as 10 most relevant proinflammatory cytokines. Intestinal tissue (colon mucosa) from RA patients $(n=10)$ and joint lysate from collagen-induced arthritis mouse model $(n=24)$. All samples were analyzed by citrulline specific sample preparation and high-end mass spectrometric analysis [1,2]. Follow-up studies were performed by multiple techniques including confocal microscopy and cell-free DNA measurement. 
Results: The proteome analysis of SF allowed deepest proteome analysis so far (Proteoforms $>1300$ ) as well as a number of citrullinated sites. The three patient groups could be differentiated by cluster analysis and the occupancy of each modified site calculated. The investigation of the intestinal tissue enabled identification of 223 citrullinated peptides from 121 proteins. Three of the peptides were unique to RA. The list of citrullinated proteins was enriched in extracellular and membrane proteins and included known targets of anticitrullinated protein antibodies (ACPAs). Investigation of collagen-induced arthritis mouse model enabled differential analysis of the proteome as response to treatment and determination of the PTMs associated with this model.

Conclusions: Our deep proteome based analysis of tissue and biofluids have enabled an extended catalogue of citrullinated proteins and sites relevant to improved disease subtyping as well as a source of citrullinated sites for future studies.

References:

[1] Bennike, T.B., Ellingsen, T., Glerup, H., Bonderup, O.K., Carlsen, T.G., Meyer, M.K., Bøgsted, M., Christiansen, G., Birkelund, S., Andersen, V., et al. (2017). Proteome Analysis of Rheumatoid Arthritis Gut Mucosa. J. Proteome Res. 16, 346-354.

[2] Tue Bennike, Kasper B. Lauridsen, Michael Kruse Olesen, Vibeke Andersen, Svend Birkelund and Allan Stensballe (2013). Optimizing the Identification of Citrullinated Peptides by Mass Spectrometry: Utilizing the Inability of Trypsin to Cleave after Citrullinated Amino Acids. Journal of Proteomics \& Bioinformatics 06.

Disclosure of Interest: None declared

DOI: 10.1136/annrheumdis-2017-eular.6934

\section{SAT0021 HUMAN CD4 T CELLS AND SYNOVIAL FIBROBLASTS COOPERATE TO PROMOTE INFLAMMATION IN THE RA SYNOVIAL JOINT}

A. Petrasca ${ }^{1}$, G. Jameson ${ }^{1}$, T. McGarry ${ }^{2}$, D.J. Veale ${ }^{3}$, U. Fearon ${ }^{2,3}$, J.M. Fletcher ${ }^{1,2} .{ }^{1}$ School of Biochemistry and Immunology; ${ }^{2}$ School of Medicine, Trinity Biomedical Sciences Institute, Trinity College Dublin; ${ }^{3}$ Department of Rheumatology, St. Vincent's University Hospital, Dublin, Ireland

Background: Rheumatoid arthritis (RA) is a chronic autoimmune disease characterised by synovial tissue proliferation and degradation of articular cartilage. Activated synovial fibroblasts proliferate and express matrix-degrading proteases, adhesion molecules and proinflammatory cytokines, which contribute to cartilage and joint destruction. Moreover, synovial cell activation correlates with infiltration of inflammatory lymphocytes and monocytes which in turn contribute to synoviocyte activation, thus further exacerbating inflammation.

Objectives: The functional relationship linking fibroblasts and $\mathrm{T}$ lymphocytes in this complex microenvironment has yet to be characterised. Therefore, we established an in vitro model to examine the outcomes of co-culturing activated human CD4 T cells with RA synovial fibroblasts.

Methods: Co-culture assays were carried out using immortalised K4IM RA synovial fibroblasts or synovial fibroblast cells derived from arthroscopy biopsies of RA patients. Human CD4 T cells were stained with a proliferation-tracking dye and co-cultured with pre-seeded synovial fibroblasts for 5 days. The resulting cell cultures and supernatants were examined for proliferation, cytokine production, secretion of matrix metalloproteinases and expression of adhesion molecules.

Results: We found that CD4 T cells and K4IM cells reciprocally induced an increased expression of adhesion molecules ICAM and VCAM. Furthermore, co-culture of CD4 T cells and synovial fibroblasts resulted in proliferation of CD4 T cells expressing increased levels of the proinflammatory cytokines IFN- $\gamma$ and IL17a and RANKL after 5 days. Lastly, co-culture of $T$ cells and synovial fibroblasts resulted in secretion of IL-6, IL-8, IFN- $\gamma$ and IL-17a and matrix metalloproteinases MMP-1 and MMP-3.

Conclusions: These results indicate that CD4 T cells work mutually with synoviocytes to create an inflammatory microenvironment likely to promote joint destruction. Future studies will characterise the role of glucose metabolism in these cells and investigate if metabolism is intrinsically coupled to effector functions in these cells.

Disclosure of Interest: None declared

DOI: 10.1136/annrheumdis-2017-eular.6294

\section{SAT0022 EPIGENOME-WIDE ASSOCIATION STUDY OF RHEUMATOID ARTHRITIS IDENTIFIES DIFFERENTIALLY METHYLATED LOCI IN B CELLS}

A. Julià ${ }^{1}$, D. Absher ${ }^{2}$, M. López-Lasanta ${ }^{1}$, N. Palau ${ }^{1}$, A. Pluma ${ }^{1}$, L. Waite Jones $^{2}$, J.R. Glossop ${ }^{3}$, W.E. Farrell ${ }^{3}$, R.M. Myers ${ }^{2}$, S. Marsal ${ }^{4} .{ }^{1}$ Grup de Recerca de Reumatologia, Vall Hebron Research Institute, Barcelona, Spain; ${ }^{2}$ Absher Lab, HudsonAlpha Institute for Biotechnology, Huntsville, United States; ${ }^{3}$ Keele University, Institute for Science and Technology in Medicine,

Staffordshire, United Kingdom; ${ }^{4}$ Grup de Recerca de Reumatologia, Vall Hebron Research Institute, Alabama, Spain

Background: Epigenetic regulation of immune cell types could be critical for the development and maintenance of autoimmune diseases like Rheumatoid Arthritis (RA). B cells are highly relevant in RA, since patients express autoantibodies and depleting this cell type is a successful therapeutic approach. Epigenetic variation, such as DNA methylation, may mediate the pathogenic activity of $B$ cells.

Objectives: In this study, we performed an epigenome-wide association study (EWAS) for RA with three different replication cohorts, to identify disease-specific alterations in DNA methylation in B cells.

Methods: Genomic methylation in isolated B lymphocytes was assayed on the Illumina HumanMethylation450 BeadChip, assaying $>450,000$ different $\mathrm{CpG}$ sites. Differential methylated positions (DMPs) were identified in a discovery cohort using a single-point analysis using logistic regression, as well as a pathway-level analysis using a newly developed permutation-based method. A discovery cohort of 50 RA patients and 75 healthy controls from Spain was used to identify the most differentially methylated regions after multiple test correction. Using an independent sample of 15 patients and 15 controls from the same population we performed a replication analysis of the most significant CpG sites and pathways. Using an additional case-control sample of 24 individuals from the UK we provided further evidence of association of the DMPs with RA. Finally, in silico datta from a cohort of systemic lupus erythematosus patients (SLE, $n=47)$ and controls $(n=56)$ from the US, we tested the association of the associated DMPs.

Results: A total of $64 \mathrm{CpG}$ sites were found to be differentially methylated in RA patients compared to controls in the discovery stage after multiple test correction $(q<0.05)$. Six biological pathways were also differentially methylated in RA B cells. Analysis of these epigenetic changes in the independent Spain cohort replicated the association of $10 \mathrm{CpG}$ sites located on 8 genes and 2 intergenic regions. Differential methylation at the $C B L$ signaling pathway was also replicated. Using the UK case-control cohort, association between RA risk and methylation levels at $C D 1 C\left(P=2.26 \times 10^{-9}\right)$ and TNFSF10 $\left(P=1.67 \times 10^{-8}\right)$ loci was further validated. Most of the replicated DMPs associated with RA were also found to be associated with differential methylation in SLE B cells.

Conclusions: Our results highlight genes that may drive the pathogenic activity of $B$ cells in RA and suggest shared methylation patterns with SLE.

Disclosure of Interest: None declared

DOI: 10.1136/annrheumdis-2017-eular.2338

\section{SAT0023 DIRECT-ACTING ANTIVIRAL-BASED THERAPY RESTORES IMMUNE TOLERANCE IN HEPATITIS C-INDUCED CRYOGLOBULINEMIA VASCULITIS}

C. Comarmond ${ }^{1}$, M. Garrido ${ }^{2}$, A.-C. Desbois ${ }^{2}$, M. $^{2}$ Costopoulos $^{3}$, M. Le Garff-Tavernier $^{3}$, S.N. Si Ahmed ${ }^{4}$, L. Alric ${ }^{5}$, H. Fontaine $^{6}$, B. Bellier ${ }^{7}$, A. Maciejewski ${ }^{7}$, M. Rosenzwajg ${ }^{7}$, D. Klatzmann ${ }^{7}$, L. Musset ${ }^{8}$, T. Poynard ${ }^{9}$, P. Cacoub ${ }^{10}$, D. Saadoun ${ }^{10} .{ }^{1}$ Internal Medicine and Clinical Immunology; ${ }^{2}$ INSERM, UMR s 959, FRE3632, Hôpital Pitié-Salpêtrière; ${ }^{3}$ Biological Hematology, Groupe Hospitalier Pitié-Salpêtrière, Paris; ${ }^{4}$ Hepatology, Hôpital d'Orléans, Orléans; ${ }^{5}$ Internal Medicine, Centre hospitalier universitaire Purpan, Toulouse; ${ }^{6}$ Hepatology, CHU Cochin; ${ }^{7}$ INSERM, UMR s 959, FRE3632; ${ }^{8}$ Immunology, UF d'Immunochimie et d'autoimmunité; ${ }^{9}$ Hepatology; ${ }^{10}$ Internal Medicine and Clinical Immunology, CHU Pitié-Salpêtrière, Paris, France

Objectives: Interferon-free direct-acting antiviral (DAA)-based therapy has proven to be very effective in patients with hepatitis $C$ virus-cryoglobulinemia vasculitis (HCV-CV). However, their mechanisms of action and their effects on cellular immunity remain poorly defined.

Methods: $27 \mathrm{HCV}-\mathrm{CV}$ patients treated with DAA therapy, 12 healthy donors (HD) and $12 \mathrm{HCV}$ were included. We investigated the effects of DAA-based therapy on cellular and cytokine abnormalities in HCV-CV patients by flow cytometry, cytokine Multiplex and enzyme-linked immunosorbent assay.

Results: Compared with HD and HCV, pre-DAA abnormalities in HCV-CV patients included a decreased percentage of $\mathrm{CD} 4^{+} \mathrm{CD} 25^{\text {hi }} \mathrm{FoxP}^{+}$regulatory $T$ cells $(P<0.01)$ with increases in $\operatorname{lgM}^{+} C D 21^{- \text {llow }}$ memory $B$ cells $(P<0.05)$, $\mathrm{CD}^{+} \mathrm{IFN} \gamma^{+}(\mathrm{P}<0.01), \mathrm{CD}^{+} \mathrm{IL} 17 \mathrm{~A}^{+}(\mathrm{P}<0.01)$ and $\mathrm{CD} 4^{+} \mathrm{CXCR5} 5^{+} \mathrm{IL} 21^{+}$follicular helper $T$ cells $(T f h)(P<0.01)$. IgM ${ }^{+} C D 21^{-1 / l o w}$ memory $B$ cells were negatively correlated with regulatory $T$ cells (Tregs) $(\mathrm{P}=0.03)$, and positively correlated with Tfh $(P=0.03)$ and serum cryoglobulin levels $(P=0.01)$. DAA-based therapy was associated with an increase in Tregs frequency $(1.5 \% \pm 0.18 \%$ versus $2.1 \%$ $\pm 0.18 \%$ ), and decreased $\operatorname{lgM}{ }^{+} \mathrm{CD} 21^{-1 / 0 w}$ memory $\mathrm{B}$ cells and Tfh percentage $(35.7 \% \pm 6.1 \%$ versus $14.9 \% \pm 3.8 \%$, and $12 \% \pm 1.3 \%$ versus $8 \% \pm 0.9 \%$, respectively). B lymphocyte stimulator receptor 3 and programmed death-ligand 1 staining expression on B cells increased in HCV-CV after DAA-based therapy (MFI $37 \pm 2.4$ versus $47 \pm 2.6, P<0.01$; and $29 \pm 7.3$ versus $48 \pm 9.3, P<0.05$ respectively). Conclusions: Our results indicate that DAA-based therapy effectively normalizes many of the disturbances in peripheral B and T lymphocyte homeostasis of HCV-CV patients.

Disclosure of Interest: None declared

DOI: 10.1136/annrheumdis-2017-eular.3911

\section{SAT0024 ALTERATIONS OF PERIPHERAL BLOOD B-CELL SUBSETS IN EARLY RHEUMATOID ARTHRITIS}

E. Suponitskava, A. Avdeeva, A.A. Aleksankin, E. Gerasimova, E. Aleksandrova, T. Popkova, A.N. Novikov, D.K. Karateev. Institute of Rheumatology, Moscow, Russian Federation

Background: Alterations of B-cell subpopulation have been described in the 